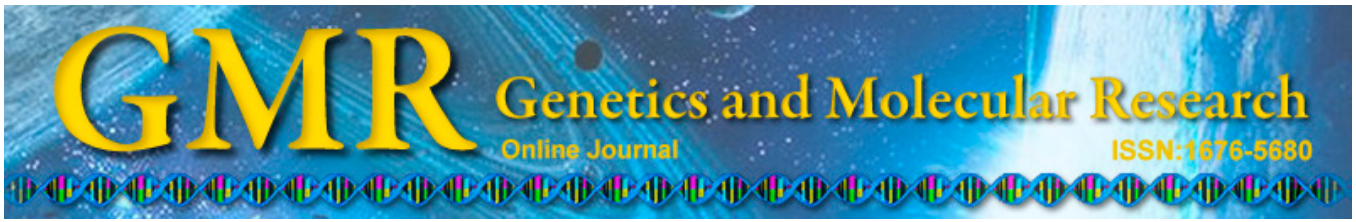

\title{
Syndromic and non-syndromic forms of retinitis pigmentosa: a comprehensive Italian clinical and molecular study reveals new mutations
}

\author{
C.O. Pierrottet ${ }^{1}$, M. Zuntini ${ }^{2}$, M. Digiuni ${ }^{1}$, I. Bazzanella ${ }^{2}$, P. Ferri' ${ }^{1}$, \\ R. Paderni ${ }^{1}$, L.M. Rossetti ${ }^{1}$, S. Cecchin ${ }^{2}$, N. Orzalesi ${ }^{1}$ and M. Bertelli ${ }^{2}$ \\ ${ }^{1}$ Department of Ophthalmology, San Paolo Hospital, University of Milan, \\ Milan, Italy \\ ${ }^{2}$ MAGI Human Medical Genetics Institute, Rovereto, Italy \\ Corresponding author: C.O. Pierrottet \\ E-mail: chiarapierrottet@tiscali.it
}

Genet. Mol. Res. 13 (4): 8815-8833 (2014)

Received October 16, 2013

Accepted February 27, 2014

Published October 27, 2014

DOI http://dx.doi.org/10.4238/2014.October.27.23

\begin{abstract}
Mutations in more than 60 different genes have been associated with non-syndromic and syndromic retinitis pigmentosa (RP), a heterogeneous group of inherited retinal dystrophies. To increase the understanding of the molecular epidemiology of the disease in Italy, we analyzed 56 patients with syndromic and non-syndromic forms of RP attending the Retinitis Pigmentosa Center of San Paolo Hospital (Milan, Italy). Patients underwent detailed clinical examination. Genomic DNA isolated from peripheral blood samples was screened for mutations in different genes according to RP form by direct sequencing analysis. The impact of novel missense mutations on protein functions was predicted by in silico analysis and protein sequence alignment. Cosegregation analysis was performed between available family members. Forty-one of the 56 probands analyzed had non-syndromic and 15 had syndromic RP forms. Putative disease-causing mutations were identified in 19 of 56 unrelated RP
\end{abstract}


probands. Mutation screening identified a total of 22 different heterozygous variants. Notably, 12 of these putative pathogenic mutations have not been previously reported. New variants were found to be located on the $U S H 2 A$, $R P G R, E Y S$, and $R H O$ genes. All 3 new variants detected in X-linked RP probands were confirmed in other affected family members. We found a positivity rate of $24.4 \%$ and $60 \%$ for probands with non-syndromic and syndromic RP, respectively. This is the first report of $R P G R \mathrm{X}$-linked RP proband-ORF15 mutations in Italian patients with X-linked (XL)-RP. In addition, this is the first report of data regarding the association between EYS mutations and non-syndromic RP forms in the Italian population.

Key words: Bardet-Biedl syndrome; Molecular screening; Retinitis pigmentosa; Usher syndrome

\section{INTRODUCTION}

Retinitis pigmentosa (RP; OMIM 268000) is a heterogeneous group of inherited retinal dystrophies characterized by photoreceptor and retinal pigment epithelium abnormalities that lead to progressive retinal degeneration, atrophy, and vision loss (Chang et al., 2011). $\mathrm{RP}$ is one of the leading causes of blindness in adults, with a prevalence of approximately 1 in 3500-5000 individuals worldwide (Rivolta et al., 2002). Typical symptoms include night blindness, progressive peripheral visual field loss (often leading to tunnel vision), and, in later stages, visual acuity impairment. Clinical hallmarks include an abnormal fundus with bone-spicule pigment deposits and attenuated retinal vessels, abnormal, diminished, or absent electroretinographic findings, and reduced visual fields. Symptoms typically start during the early teenage years, and severe visual impairment occurs by $40-50$ years of age. There are also early-onset, late-onset, and even nonpenetrant forms of RP (Daiger et al., 2007).

RP can be subdivided into non-syndromic RP, without systemic abnormalities, and syndromic RP, in which retinal degeneration is associated with other symptoms due to systemic diseases. The most frequent syndromic form of RP is Usher syndrome (OMIM \#276900-902), which manifests as early-onset or congenital neurosensory hearing impairment followed by development of RP (Kimberling et al., 2000; Keats and Savas, 2004). The second most common syndromic form is Bardet-Biedl syndrome (OMIM \#209900), which includes RP, polydactyly, obesity, renal abnormalities, hypogenitalism, and mental retardation (Katsanis, 2004). Many other complex, pleiotropic conditions include RP as a component (Daiger et al., 2007).

Mutations in more than 60 different genes associated with non-syndromic and syndromic RP phenotypes have been described (RetNet, 2013 database: https://sph.uth.edu/retnet/).

Non-syndromic RP can be inherited in an autosomal dominant (adRP), recessive (arRP), or X-linked (XL-RP) manner. Rare cases of RP presenting mitochondrial (Mansergh et al., 1999) or digenic inheritance (Kajiwara et al., 1994), uniparental disomy, and incomplete penetrance (Rivolta et al., 2002) have also been described. A number of cases cannot be classified and are therefore referred to as isolated or sporadic RP (sRP; single occurrence in a family) (Pagon and Daiger, 2000).

adRP accounts for $15-25 \%$ of all RP cases (Pagon and Daiger, 2000; Daiger et al., 2007), depending on the country and the ethnic group analyzed. Two studies reported a prevalence of approximately $17 \%$ for adRP in the Italian population (Fossarello et al., 1993; Ziviello 
et al., 2005), which agrees with the results of other studies carried out in Southern Europe (Ayuso et al., 1995). The genes typically associated with adRP include RHO (25-30\%), PRPF31 (15-20\%), PRPH2 (5-10\%), RPI (5-10\%), PRPF3, and IMPDH1 (3-5\%) (Pagon and Daiger, 2000; Fahim et al., 2013).

arRP is estimated to account for 5-20\% of all forms of non-syndromic RP (Pagon and Daiger, 2000; Daiger et al., 2007). The genes most frequently affected in arRP patients include EYS and RPE65 (Pagon and Daiger, 2000).

In addition, 5-15\% of RP patients show X-linked inheritance (XL-RP) (Pagon and Daiger, 2000), which is generally the most severe form, characterized by early onset and rapid progression. Two genes, $R P 2$ and $R P G R$, account for $10-20$ and $70-90 \%$ of cases, respectively (Vervoort et al., 2000; Fahim et al., 2013).

The high degree of genotypic and phenotypic variability, including within families, as well as the current lack of specific treatments, make customized management necessary. These treatments should consider clinical, genetic, rehabilitative, and psychological aspects (Parmeggiani et al., 2011). In this context, accurate molecular screening enables genotypephenotype correlation studies, which are useful for predicting disease severity and for effective genetic counseling. Moreover, since gene therapy prospects have improved for a variety of retinal disorders, including RP (Liu et al., 2011; Beltran et al., 2012), genetic screening results can provide important information that can be used to develop innovative treatments.

Here, we present the results of clinical and genetic characterization of 56 Italian probands with syndromic and non-syndromic forms of RP, contributing to the elucidation of RP molecular epidemiology in Italy. We identified a total of 22 different causative mutations in 19 patients and describe 12 new mutations that were further characterized for their putative pathogenic potential.

\section{MATERIAL AND METHODS}

\section{Subjects and clinical evaluation}

Fifty-six consecutive probands with clinically suspected RP and their family members were examined at the University Eye Clinic, Health Department, San Paolo Hospital (Milan, Italy) from May 2011 to October 2012. All RP families were from Northern Italy. A team of ophthalmologists and medical geneticists (the latter from MAGI Human Medical Genetics Institute, Rovereto, Italy) evaluated all patients for diagnostic purposes, including which form of RP was to be confirmed at the molecular level. All subjects enrolled in the study underwent comprehensive ophthalmic examination, including slit-lamp examination, tonometry, and best-corrected visual acuity as evaluated using Early Treatment Diabetic Retinopathy Study charts and procedures. Goldmann kinetic perimetry (Goldmann Perimeter; Haag Streit AG; Mason, OH, USA) with a V4e target was performed on all patients. An I4e target was used to provide greater detail regarding the visual fields of patients. Fundus autofluorescence, infrared reflectance retinography, optical coherence tomography (Spectralis HRA-OCT; Heidelberg Engineering; Heidelberg, Germany), and electroretinograms following the guidelines of the International Society for Clinical Electrophysiology of Vision (MonPackOne; Metrovision; France), were performed in all patients. Demographic details, age of onset, and disease duration were recorded, along with all available information on personal and familial medical history, which was used to determine the type of genetic inheritance and to draw pedigrees. 
The study followed institutional guidelines and the Declaration of Helsinki. Patients enrolled in the study signed 2 written informed consent forms, one for genetic testing and the other for publication of clinical and genetic data for research.

\section{Mutation screening}

Peripheral blood samples obtained from patients and available family members were sent to the MAGI Laboratory (MAGI Human Medical Genetics Institute, Rovereto, Italy) for isolation of DNA and genetic testing. Genomic DNA was extracted using the Gentra Puregene Blood Kit (Qiagen; Hilden, Germany) according to manufacturer instructions. DNA integrity and quality were evaluated by agarose gel electrophoresis and with a BioSpec-Nano spectrophotometer (Shimadzu Italia; Milan, Italy), respectively, prior to analysis.

Specific protocols for direct sequencing of each form of RP were previously optimized at the MAGI Laboratory. A total of 13 genes (Table 1) to be analyzed for different syndromic and non-syndromic forms of RP were selected based on mutation prevalence rates reported in the literature and on international guidelines (Pagon and Daiger, 2000; Waters and Beales, 2003; Fahim et al., 2013).

All coding regions and the intron/exon junctions of genes selected were amplified by polymerase chain reaction (PCR), purified, and analyzed using a Beckman Coulter CEQ 8000 sequencer (Beckman Coulter; Milano, Italy). PCR primer sequences and conditions are available upon request.

\begin{tabular}{lll}
\multicolumn{2}{c}{ Table 1. Genes analyzed for each form of retinitis pigmentosa. } & \\
\hline Gene & Reference sequence (GenBank) & Disease \\
\hline$R H O$ & NM_000539.3 & adRP, sRP \\
$P R P H 2$ & NM_000322.4 & adRP, sRP \\
$R P 1$ & NM_006269.1 & adRP \\
$P R P F 3$ & NM_004698.1 & adRP \\
$P R P F 31$ & NM_015629.3 & adRP \\
$I M P D H 1$ & NM_000883.3 & adRP \\
$R P E 65$ & NM_000329.2 & arRP \\
$E Y S$ & NM_001142800.1 & arRP, sRP \\
$R P 2$ & NM_006915.1 & XL-RP \\
$R P G R$ & NM_000328.2 & XL-RP \\
$R P G R-O R F 15$ & NM_001034853.1 & XL-RP \\
USH2A & NM_007123.4 & USHER 2A \\
$B B S 1$ & NM_024649.4 & BBS \\
BBS10 & NM_024685.3 & BBS \\
\hline
\end{tabular}

\section{Mutation and bioinformatic analysis}

The electropherograms of all fragments amplified were analyzed using ChromasPro 1.5 (Technelysium Pty Ltd.; Australia) and Sequencher 5.0 (Gene Codes ${ }^{\circledR}$; Ann Arbor, MI, USA) software and compared to GenBank reference sequences (Table 1) using the Basic Local Alignment Search Tool (BLAST; http://blast.ncbi.nlm.nih.gov). All mutations detected were sequenced bidirectionally at least twice for confirmation. DNA mutation nomenclature reflects cDNA numbering with +1 corresponding to the A of the ATG translation initiation codon in the reference sequence. 
Sequence variations that were ambiguously reported in the literature and/or in dbSNP (http://www.ncbi.nlm.nih.gov/SNP/) as benign polymorphisms were further analyzed for their putative pathogenicity. To identify mutations previously reported as pathogenic, the Human Gene Mutation Database (http://www.hgmd.cf.ac.uk) and Retina International database (http://www.retina-international.org) were consulted. Missense mutations were further investigated using MutationTaster (http://www.mutationtaster.org), Sorting Intolerant From Tolerant (http://sift.jcvi.org/), and Polyphen-2 (Polymorphism Phenotyping v2; http://genetics.bwh. harvard.edu/pph2) algorithms to predict their functional impact. Nonsense, frameshift, and canonical splice site variants were considered to be pathogenic.

All potentially pathogenic variants were detected by direct sequencing of 100 healthy subjects from the same geographic area. When available, DNA samples from family members were analyzed to confirm any cosegregation of the mutation with the phenotype.

\section{RESULTS}

\section{Patients included in the study}

Of the 56 unrelated probands enrolled in the study, 41 (73.2\%) had non-syndromic RP and 15 (26.8\%) had syndromic forms. Among patients with non-syndromic RP, 23 (56.1\%) were familial cases and $18(43.9 \%)$ were sporadic cases. Pedigree analysis of patients with inherited forms of the disease led to the classification of 9 adRP cases, 6 arRP cases, and 8 probands with XL-RP. Among patients with syndromic disease, 13 (86.7\%) were classified as Usher2A and the remaining $2(13.3 \%)$ were classified as having Bardet-Biedl syndrome.

\section{Mutation screening}

Putative disease-causing mutations were identified in 19 of the 56 unrelated RP probands analyzed in this study (33.9\% of cases). Mutation frequencies were $22.2 \%$ (2 mutation carriers in 9 patients; $2 / 9)$ for adRP; $16.7 \%$ for arRP (1/6) and sporadic patients $(3 / 18)$, $50 \%$ for XL-RP (4/8) and patients with Bardet-Biedl syndrome (1/2), and 61.5\% (8/13) for Usher2A patients.

We identified a total of 22 different heterozygous variants not previously reported in the literature and/or in dbSNP as benign polymorphisms, which were absent in the 100 control subjects. These included 10 missense mutations, 7 frameshift mutations, 2 nonsense mutations, 2 splicing mutations, and 1 variant in a regulatory region. Eight of 22 sequence variations were observed in the USH2A gene, 4 in the RPGR gene ( 3 of these in the ORF15 exon), 5 in EYS, 2 in RHO, 1 in the PRPH2 gene, and 2 in the Bardet-Biedl-associated BBS10 gene.

Twelve of these putative pathogenic mutations ( $54.5 \%$ of identified variants) are reported for the first time (Table 2), while 10 have been previously described in RP patients (Table 3). The new variants are located in USH2A (3 mutations), RPGR (3), EYS (5), and RHO (1).

The nonsense and the 3 frameshift variants were considered to be pathogenic. To predict the impact on protein function of the 7 missense mutations, we performed in silico analysis using 3 different algorithms. For 5 of these mutations (p.Gly182Val in RHO; p.Cys2139Arg and p.Cys2927Arg in EYS; p.Gly148Val in RPGR; p.Cys691Arg in USH2A; Table 2), at least 2 bioinformatic tools predicted the variants to be deleterious and the mutations were therefore considered to be pathogenic. The $U S H 2 A$ variant p.Asn1313Ser was evaluated as pathogenic 
only by MutationTaster, while the p.Pro1631Ser missense variant in EYS was unequivocally predicted to be benign.

\begin{tabular}{|c|c|c|c|c|c|c|c|}
\hline Proband & Phenotype & Gene & Nucleotide change & Protein effect & SIFT & Mutation Taster & PolyPhen2 \\
\hline $\mathrm{P} 2[\mathrm{~F} 2]$ & $\operatorname{adRP}$ & RHO & c. $545 \mathrm{G}>\mathrm{T}$ & p.Gly182Val & $\mathrm{P}^{*}[0]$ & $\mathrm{P}[0.99]$ & $\operatorname{Prob}^{\#}[1]$ \\
\hline \multirow{2}{*}{ P15 [F3] } & arRP & EYS & c. $-459 \mathrm{C}>\mathrm{T}$ & Unknown & - & - & - \\
\hline & $\operatorname{arRP}$ & EYS & c.9299_9302del & p.Thr3100Lysfs*26 & - & - & - \\
\hline \multirow[t]{2}{*}{ P24 } & $\mathrm{sRP}$ & EYS & c. $4891 \bar{C}>\mathrm{T}$ & p.Pro1631Ser & $\mathrm{B}^{\dagger}[0.06]$ & $\mathrm{B}[0.99]$ & $\mathrm{B}[0.12]$ \\
\hline & sRP & $E Y S$ & c. $6415 \mathrm{~T}>\mathrm{C}$ & p.Cys2139Arg & $\mathrm{P}[0]$ & B [0.99] & Prob [0.97] \\
\hline P33 & sRP & $E Y S$ & c. $8779 \mathrm{~T}>\mathrm{C}$ & p.Cys2927Arg & $\mathrm{P}[0]$ & $\mathrm{B}[0.85]$ & Prob [0.99] \\
\hline P35 [F5] & XL-RP & RPGR-ORF 15 & c. $2023 \mathrm{G}>\mathrm{T}$ & p.Glu675* & - & - & - \\
\hline P36 [F6] & XL-RP & $R P G R$ & c. $443 \mathrm{G}>\mathrm{T}$ & p.Gly148Val & $\mathrm{P}[0]$ & $\mathrm{P}[0]$ & Prob [0.99] \\
\hline P39 [F11] & XL-RP & $R P G R-O R F 15$ & c.1967del & p.Asp656Valfs*41 & - & - & - \\
\hline P45 & USHER2 & $U S H 2 A$ & c. $2071 \mathrm{~T}>\mathrm{C}$ & p.Cys691Arg & $\mathrm{P}[0]$ & $\mathrm{P}[0.99]$ & Prob [0.99] \\
\hline P51 & USHER2 & $U S H 2 A$ & c.2013_2016del & p.Arg671Serfs*84 & - & - & - \\
\hline P52 [F10] & USHER2 & $U S H 2 A$ & c. $3938 \bar{A}>\mathrm{G}$ & p.Asn1313Ser & $\mathrm{B}[0.42]$ & $\mathrm{P}[0.83]$ & $\mathrm{B}[0.03]$ \\
\hline
\end{tabular}

*P $=$ pathological; ${ }^{*}$ Prob = probably pathological; $\mathrm{B}^{\dagger}=$ benign.

Table 3. List of known pathogenic mutations identified.

\begin{tabular}{|c|c|c|c|c|c|}
\hline Proband & Phenotype & Gene & Nucleotide change & Protein effect & Reference $^{\S}$ \\
\hline $\mathrm{P} 8[\mathrm{~F} 1]$ & adRP & PRPH2 & c. $493 \mathrm{~T}>\mathrm{C}$ & p.Cys165Arg & CM074464 \\
\hline $\mathrm{P} 22$ & sRP & $\mathrm{RHO}$ & c. $1040 \mathrm{C}>\mathrm{T}$ & p.Pro347Leu & CM900199 \\
\hline P38 [F4] & XL-RP & RPGR-ORF 15 & c.2405_2406del & p.Glu802Glyfs*32 & CD004115 \\
\hline \multirow[t]{2}{*}{ P43 } & BBS & $B B S 10$ & c. $1091 \overline{\mathrm{de}} \mathrm{el}$ & p.Asn364Thrfs*5 & White, 2007 \\
\hline & BBS & BBSIO & c. $1677 \mathrm{del}$ & p.Tyr559* & CD064494 \\
\hline P44 [F7] & USHER2 & USH $2 A$ & c. $2168-1 \mathrm{G}>\mathrm{C}$ & - & CS073533 \\
\hline P49 [F8] & USHER2 & USH $2 A$ & c. $2299 \mathrm{del}$ & p.Glu767Serfs*21 & CD982997 \\
\hline \multirow[t]{2}{*}{ P50 } & USHER2 & USH $2 A$ & c. $187 \mathrm{C}>\mathrm{T}$ & p.Arg63* & CM001804 \\
\hline & USHER2 & $U S H 2 A$ & c. $1663 \mathrm{C}>\mathrm{G}$ & p.Leu555Val & CM014225 \\
\hline P55 [F9] & USHER2 & USH $2 A$ & c. $2167+5 \mathrm{G}>\mathrm{A}$ & - & CS021381 \\
\hline P56 & USHER2 & $U S H 2 A$ & c.2299del & p.Glu767Serfs*21 & CD982997 \\
\hline
\end{tabular}

\$Refers to HGMD accession number (Human Gene Mutation Database; http://www.hgmd.cf.ac.uk) or "Reference" section.

\section{New variants associated with non-syndromic RP}

The age of patients with non-syndromic forms of RP ranged from 9-80 years (mean: 48 years), with a mean age at onset of 25 years. Molecular screening results were positive for 10 of 41 patients with clinical diagnosis of non-syndromic RP (24.4\%). Clinical features and mutation details for probands whose causative mutations were identified are reported in Table 4. Nine of the 12 potential mutations $(75 \%)$ identified in this screening have not been previously described.

\section{RHO p.Gly182 Val}

This mutation was detected as heterozygous in one adRP proband (P2-F2), a 40-yearold woman whose mother also had RP (Figure 1). The p.Gly182Val variant was a new missense mutation in exon 3 of the $R H O$ gene (c. $545 \mathrm{G}>\mathrm{T}$ ). The glycine residue affected was located in the extracellular domain of the protein and was found to be highly conserved in all organisms tested, suggesting that this amino acid has an important functional role (Figure 1). 


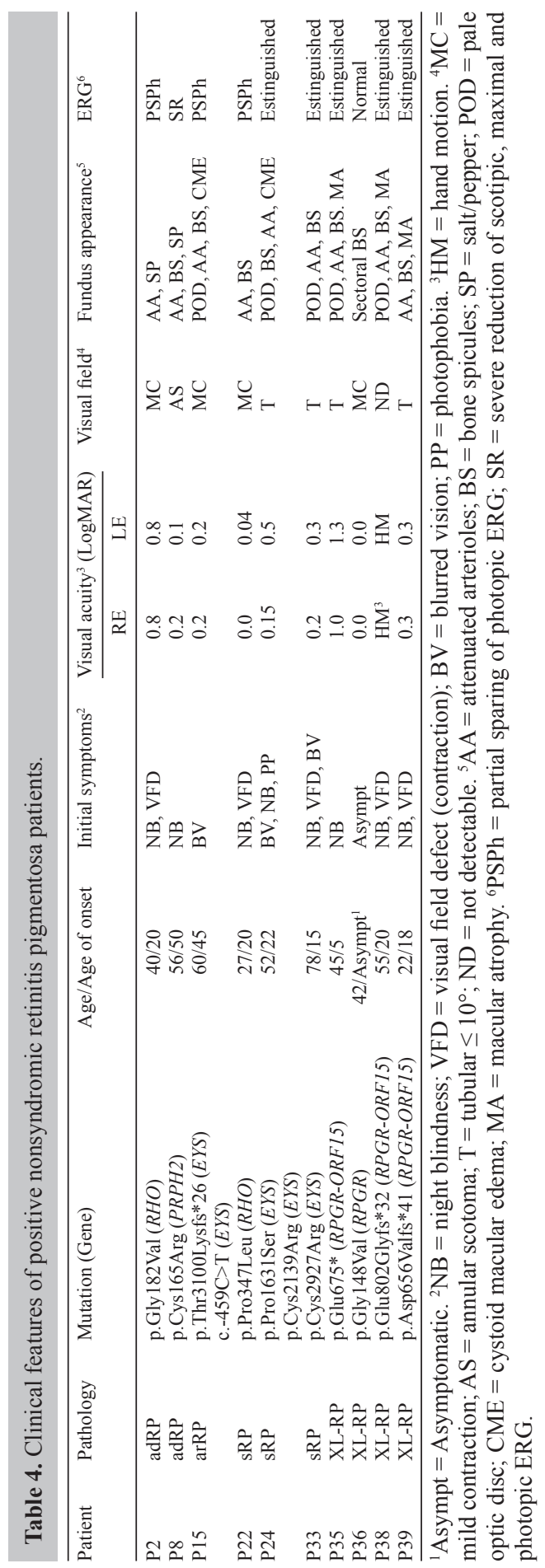


Indeed, all software used for in silico analysis predicted that the substitution affected protein function (Table 2). A pathogenic missense mutation in the same codon was previously reported by Sheffield et al. (1991) (p.Gly182Ser).
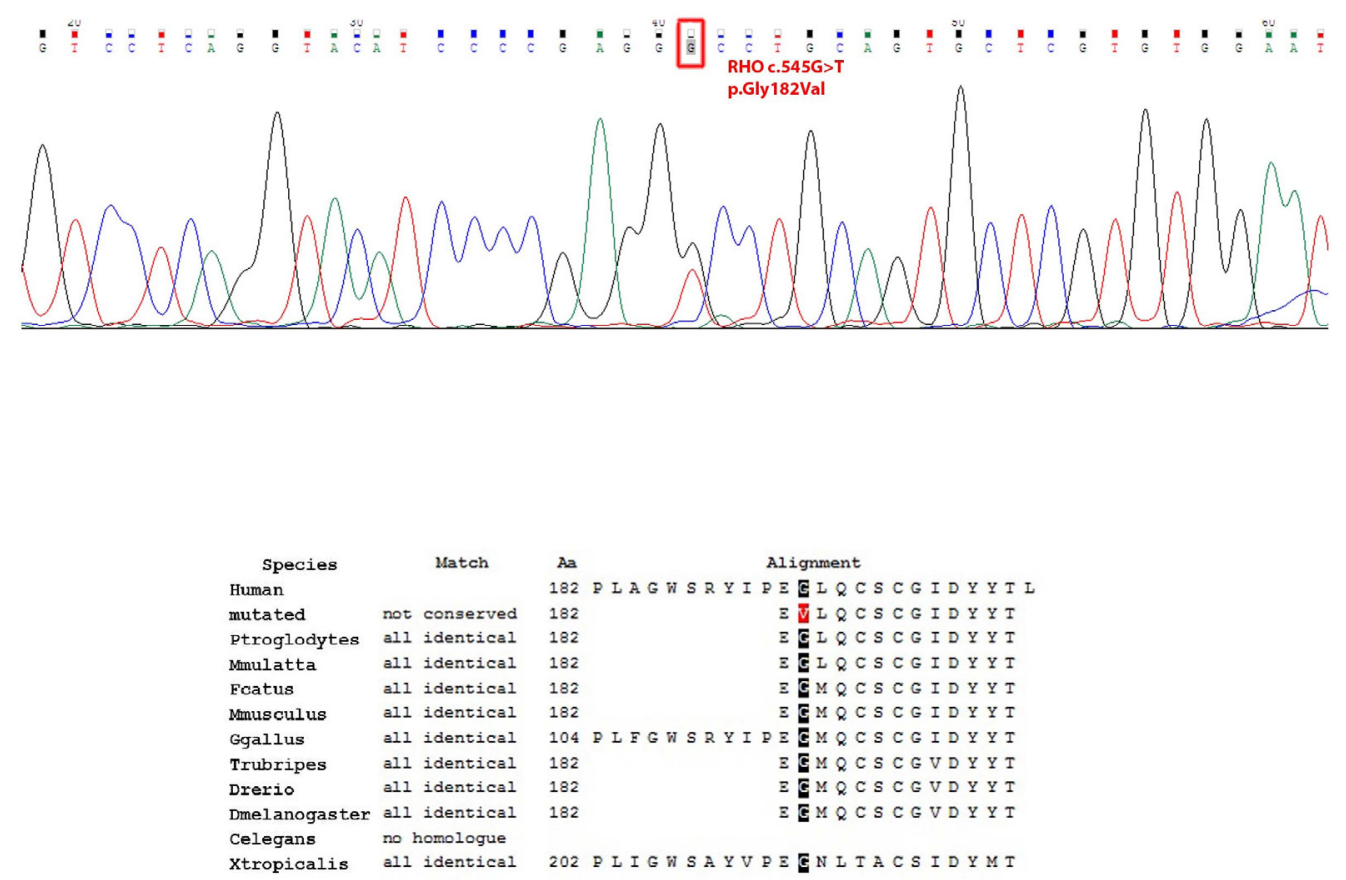

Figure 1. Sequence chromatogram of the p.Gly182Val mutation in RHO exon 3 identified in autosomal dominant $\mathrm{RP}$ patient P2. Conservation of affected amino acids across species determined by the MutationTaster tool is shown below.

\section{EYS c. $-459 C>T$ and p.Thr3100Lysfs $* 26$}

In proband P15 of family F3, a 60-year-old woman, molecular screening for genes involved in arRP (Table 1) revealed 2 different heterozygous variants in the EYS gene: a mutation in non-coding exon 1 (c.-459C $>$ T) and a small deletion (c.9299_9302del; p.Thr3100Lysfs*26). Although the effect of the first variant on protein function could not be evaluated using bioinformatic tools, it is likely to be pathogenic as 3 different genetic alterations in the same region of EYS have been described (Barragán et al., 2010). Their potential pathogenicity is thought to be related to their location in regulatory regions that are important for protein translation (Scheper et al., 2007). The second new variant detected in P15 was considered to be pathogenic because deletion of 4 nucleotides at codon 3100 in exon 43 led to a frameshift and premature protein termination ( $\mathrm{p}$. Thr3100Lysfs*26).

\section{EYS p.Pro1631Ser and p.Cys2139Arg}

The proband with these 2 missense variations (P24) was a 52-year-old woman. Since 
no other members affected were identified in the family, this case was presumed to be a sporadic case. Both putative mutations (c.4891C $>$ T, p.Pro1631Ser; c.6415T $>$ C, p.Cys2139Arg) were identified as heterozygotes in the EYS gene. Although the p.Pro1631Ser variant in exon 27 has not been reported as a polymorphism in the literature or databases and was not identified in our control samples, all prediction tools indicated it to be benign. Notably, the same variant was detected in the unaffected son of P24. Further analyses are required to clarify the potential role of this genetic variant.

The missense mutation, p.Cys2139Arg, in exon 32 causes substitution of the polar residue cysteine 2139 with the basic arginine in the epidermal growth factor (EGF)-like 21 domain. Since Cys2139 is conserved in primates but not in other species, the effect of its substitution on protein function was determined to be benign by the MutationTaster tool, while Sorting Intolerant From Tolerant and Polyphen-2 predicted the variants to be deleterious. A pathogenic missense mutation in the same codon (p.Cys2139Tyr) was described by Audo et al. (2010), thus supporting the pathogenic potential of the variant detected in P24.

\section{EYS p.Cys2927Arg}

This missense mutation (c.8779T $>\mathrm{C}$; Figure 2) was observed as a heterozygous mutation in one 78-year-old male patient (P33). The c.8779T $>\mathrm{C}$ variant was located on exon 43 in the EGF-like 26 domain and resulted in a substitution of a cysteine residue that is conserved in primates and in Caenorhabditis elegans (Figure 2). Two of the 3 bioinformatic tools predicted the variant to be deleterious, and it was therefore classified as potentially pathogenic.
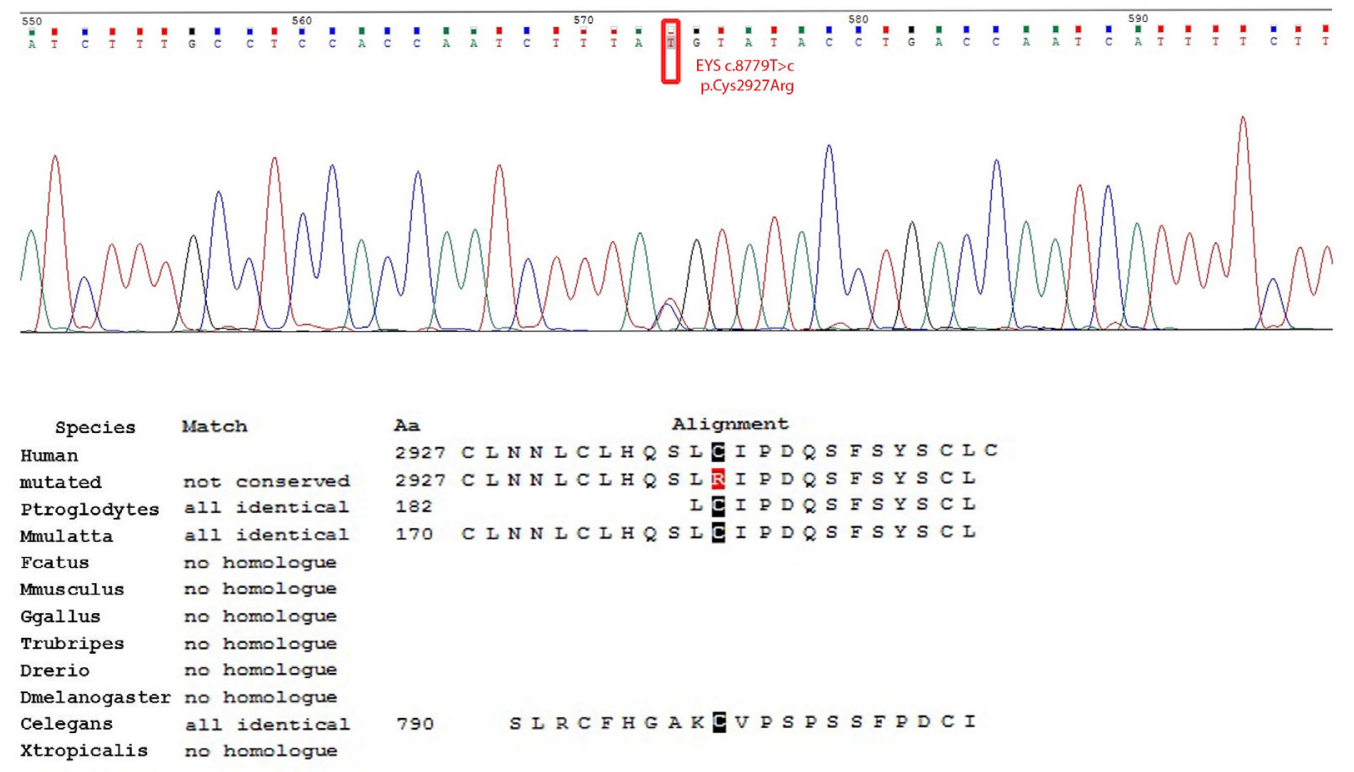

Figure 2. Sequence chromatogram of the heterozygous p.Cys2927Arg mutation in EYS identified in sporadic retinitis pigmentosa proband P33. This variant was on exon 43 in the EGF-like 26 domain and resulted in substitution of a cysteine residue that is conserved in primates and in Caenorhabditis elegans. 


\section{RPGR p.Glu675*}

This new nonsense mutation was identified in family F5, which contains 6 members with RP. Pedigree analysis suggested X-linked inheritance (Figure 3). In the proband (P35), a 45-year-old male, molecular screening identified the hemizygous nonsense variant c. $2023 \mathrm{G}>\mathrm{T}$ on the ORF15 exon of the RPGR gene, which results in the synthesis of a truncated protein (p.Glu675*) (Figure 3). In this family, the mutation segregated with the disease phenotype. Indeed, the same genetic alteration was confirmed in the affected nephew and niece of the proband (Figure 3). The proband's nephew, a 17-year-old male, first showed symptoms of night blindness at 11 years of age. The niece's proband, a 20-year-old female, had severe myopia (-8.00 sphere in both eyes), with good central visual acuity (0.0 logMAR in both eyes) and atypical retinal pigmentation adjacent to the vascular arcade. The p.Glu675* mutation was not identified in the first cousin of P35, who showed clinical signs of RP starting at 24 years of age and neurosensory hearing deficit at birth; Usher syndrome was therefore postulated for this patient.
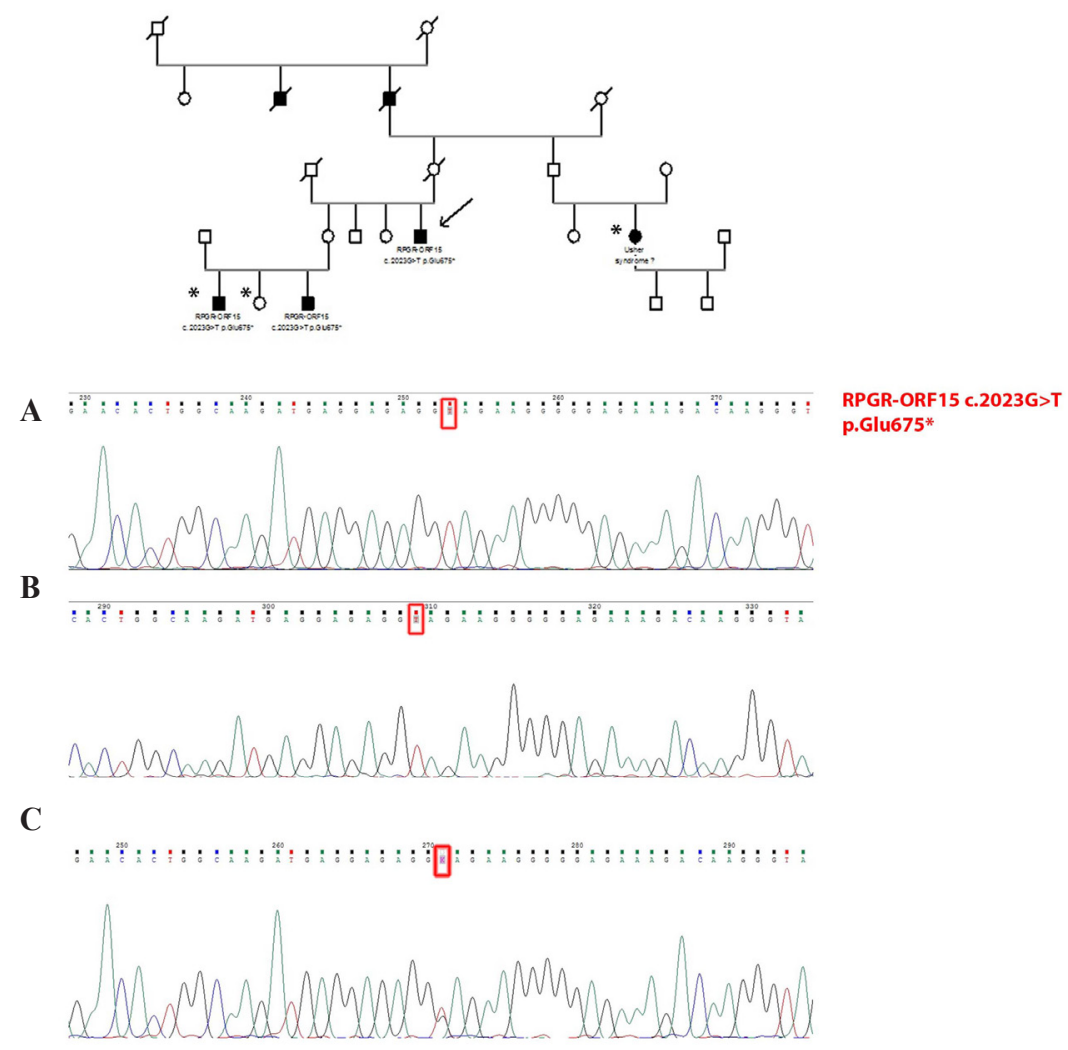

Figure 3. Pedigree of family F5 suggesting X-linked inheritance of retinitis pigmentosa. Molecular screening of proband P35 identified the new hemizygous nonsense variant p.Glu675* (c.2023G $>$ T) on the ORF15 exon of the RPGR gene (A). The same genetic alteration was confirmed in the affected nephew (B) and niece (C) of the proband. Solid symbols indicate affected individuals. Asterisks indicate family members for whom DNA was available for genomic analysis; arrow indicates the proband. 


\section{RPGR p.Gly148Val}

This new mutation was identified as a heterozygous variant in proband P36, a 42-year-old female whose mother and son also had RP (Figure 4). She was asymptomatic and had not noticed any changes in her visual field, although Goldmann perimetry showed superotemporal scotoma in the left eye. Fundus examination revealed that the retina had a salt-and-pepper pattern and bonespicule-shaped pigment changes in the inferior periphery. Electroretinography testing was normal in both eyes, with lower amplitudes in the left eye. Monolateral visual field loss corresponding to fundus abnormalities observed in P36 confirmed the clinical data of mildly affected X-linked recessive RP carriers showing phenotypic signs of the disease, depending on non-random X chromosome inactivation (Koenekoop et al., 2003). The son of P36, who was 12 years old, presented with a history of night blindness at 5 years old. The rapid progression of RP observed in this young patient indicates the severity of XL-RP in affected males, which often leads to legal blindness by the end of the 4th decade. In this family, the c.443G $>$ T mutation segregated with the RP phenotype and was detected in the mother and son of the patient P36 (Figure 4), confirming that this variant is disease-causing. This was also indicated by bioinformatic prediction of the mutation impact.

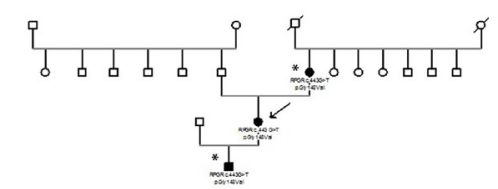

\section{A}

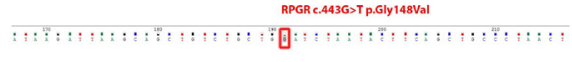

B
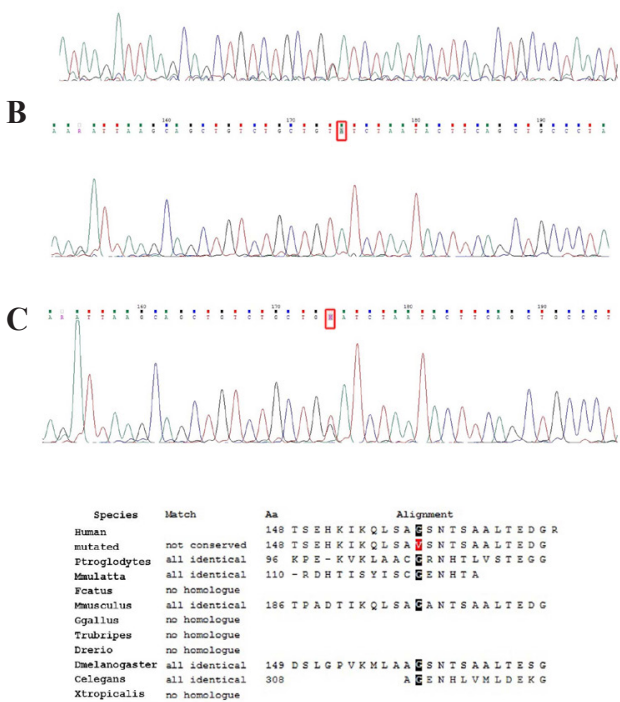

Figure 4. Pedigree of family F6 with X-linked inheritance of retinitis pigmentosa (RP). The new p.Gly148Val mutation was identified in $R P G R$ exon 5 as a heterozygous variant in proband P36 (chromatogram A), a 42-year-old female whose son and mother also had RP (chromatograms B and C, respectively). Solid symbols indicate affected individuals. Asterisks indicate family members for whom DNA was available for genomic analysis; arrow indicates the proband. 


\section{RPGR p.Asp656Valfs*41}

There were several members affected in family F11 of proband P39 (Figure 5). The proband (P39), a 22-year-old male, had night blindness for as long as he could remember, with peripheral vision problems beginning at age 14. Molecular screening for XL-RP-associated genes revealed a new frameshift variant on the ORF15 exon of the RPGR gene (c.1967del). This variant resulted in synthesis of a truncated protein and was therefore considered to be pathogenic. Sequencing analysis confirmed the same variant in the affected sister (heterozygous mutation), aunt (heterozygous mutation), and cousin (hemizygous mutation) of the proband, indicating X-linked inheritance in this family.

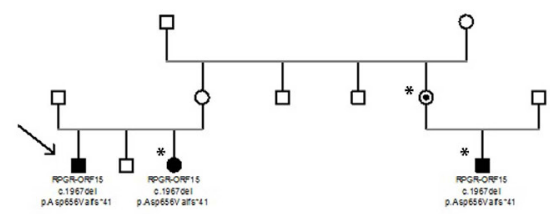

A

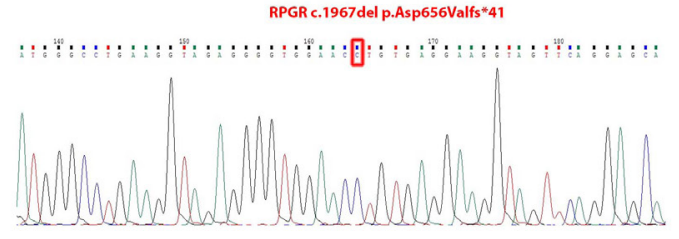

B
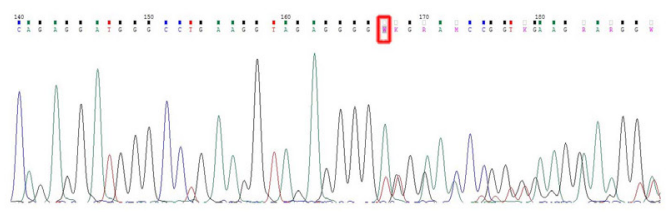

C

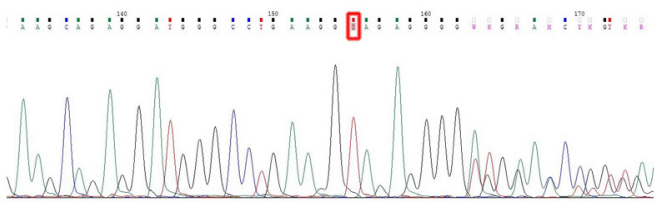

D

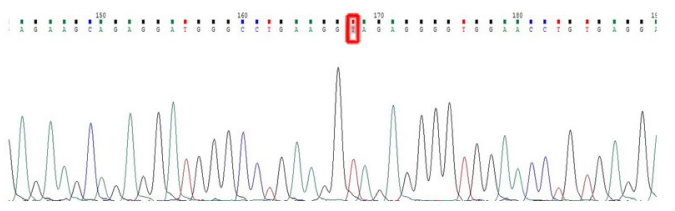

Figure 5. Pedigree of family F11. Molecular screening of patient P39 for XL-RP-associated genes revealed a new frameshift variant on the ORF15 exon of the RPGR gene (c.1967del; chromatogram A). Sequencing analysis confirmed the same variant in the affected sister (heterozygous mutation; chromatogram B), aunt (heterozygous mutation; chromatogram C), and cousin (hemizygous mutation; chromatogram D) of the proband. Solid symbols indicate affected individuals. Asterisks indicate family members for whom DNA was available for genomic analysis; arrow indicates the proband. 


\section{New variants associated with syndromic RP}

The age of patients affected by syndromic forms of RP ranged from 21-79 years (mean age: 45 years), with a mean age at onset of 16 years (range 0-31 years). Molecular screening was positive for 9 of 15 patients with clinical diagnosis of Usher2A or Bardet-Biedl syndrome ( $60 \%$ of cases). Clinical features and mutation details of probands with identified causative mutations are reported in Table 5. Three of the 8 potential mutations (37.5\%) identified in this screening have not been previously reported. All of these new variants were on the USH2A gene (Table 2).

\section{USH2A p.Cys691Arg}

This new mutation was detected in proband $\mathrm{P} 45$, a 30-year-old female patient whose familial medical history could not be ascertained. Molecular screening of the USH2A gene detected a new heterozygous variant on exon 12 (c.2071T $>$ C; p.Cys691Arg) (Figure 6). This mutation causes the substitution of a highly conserved cysteine residue with a basic arginine at codon 691 in the extracellular laminin EGF-like 3 domain of the protein. The importance of this residue is further supported by the results obtained through in silico analysis using 3 different algorithms, which univocally indicated this mutation to be pathogenic. A missense pathogenic mutation affecting the same codon has also been described (Nakanishi et al., 2009).

\section{USH2A p.Arg671Serfs $* 84$}

A new frameshift mutation involving deletion of a base on exon 12 (c.2013_2016del) was identified in proband P51, a 79-year-old woman who noted neurosensory hearing impairment at the age of 6 years with night blindness and loss of peripheral vision at 18 years. This heterozygous variant led to synthesis of a truncated protein (p.Arg671Serfs*84) and was found to be located in the extracellular laminin EGF-like 3 domain of the protein, similarly to the new variant identified in P45.

\section{USH2A p.Asn1313Ser}

A new missense mutation (c.3938A $>\mathrm{G}$ ) located on exon 18 of the USH2A gene was found to be heterozygous in proband P52, a 56-year-old woman with a family history of Usher syndrome. She presented with a 6-year history of neurosensory hearing impairment and reported dark adaptation problems and peripheral visual field problems since the age of 14 years. The c.3938A $>\mathrm{G}$ variation led to substitution of a polar asparagine residue with a serine residue at position 1313 in the extracellular fibronectin type-III 3 domain of the usherin protein. Two prediction tools indicated low pathogenic potential for this amino acid change, while MutationTaster predicted an impact on protein function, as this position is highly conserved in various organisms. 
C.O. Pierrottet et al.

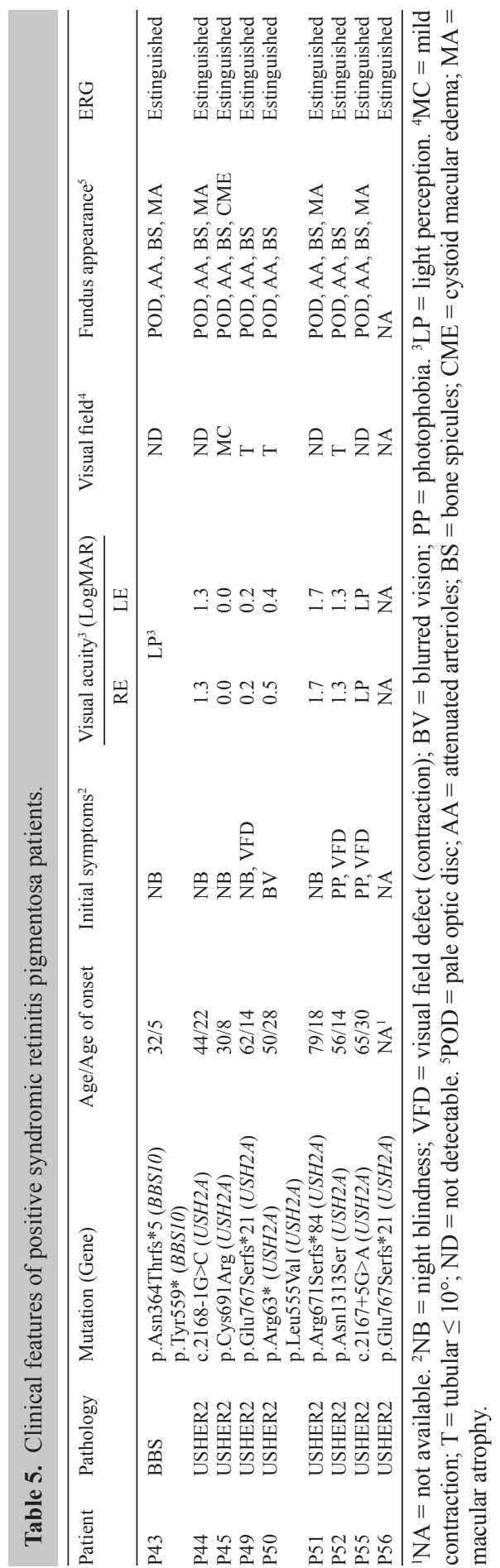


USH2A c.2071T>C p.Cys691Arg

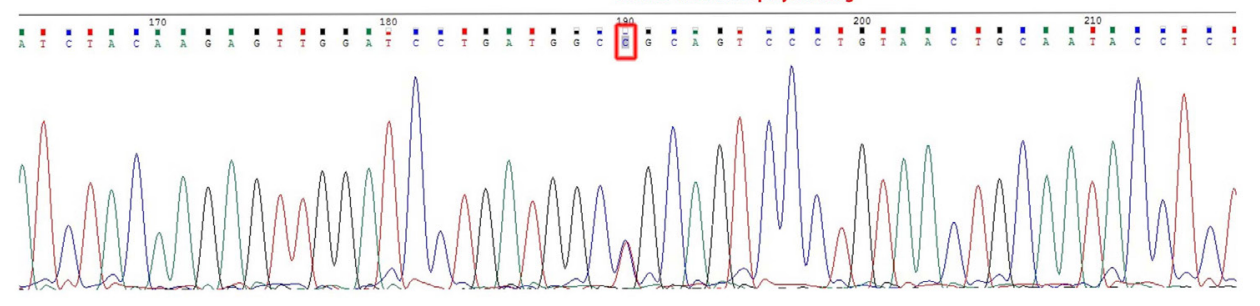

\begin{tabular}{|c|c|c|c|}
\hline Species & Match & aa & Alignment \\
\hline Human & & 691 & $\Sigma Y N I Q \equiv L D P D G \mathbb{C} S P C N C N T S G T V D$ \\
\hline mutated & not conserved & 691 & FN $L Q E L D D G$ ESPCNCNTSGTV \\
\hline Ptroglodytes & all identical & 691 & $\Xi Y N L Q \equiv L D P D G \mathrm{CS} P C N C N$ ISGTV \\
\hline Mrulatta & all identical & 690 & $\Sigma Y N L Q \equiv L D P D G \overline{\mathrm{g}} S$ PCNCNTSGIV \\
\hline Fcatus & all identical & 843 & $Y \equiv E L Q Q N N S E L$ C L P NCDKTGTV \\
\hline Mrnusculus & all identical & 688 & FYDQALDPDG $R$ PCNCN PSGIV \\
\hline Ggallus & a.1 identical & 690 & FY LQQSNPDG GSPCNCNISGTV \\
\hline Trubripes & a.1 identical & & \\
\hline Drerio & all identical & 703 & FYKLQSALADG $\mathrm{C} R$ A CNCNIAG TV \\
\hline Dmelanogas ter & all identical & 705 & WSGLKASNPNG \\
\hline Celegans & no homologue & 482 & $V A C T C N L L G T Y$ \\
\hline xtropicalis & all identical & 468 & E PCICN PIGIL \\
\hline
\end{tabular}

Figure 6. Sequence chromatogram of the new mutation p.Cys691Arg in USH2A detected in proband P45, a 30-yearold female whose familial medical history could not be ascertained. This position has been highly conserved throughout evolution, as determined using the MutationTaster tool.

\section{DISCUSSION}

In the present study, collaboration between a referral ophthalmology department and a team of geneticists with knowledge of retinal dystrophies enabled clinical and genetic characterization of an Italian case series of 56 probands with different forms of non-syndromic and syndromic RP. We enrolled all patients clinically suspected of having RP without any other selection criterion; therefore, our sample included affected subjects who report annually to our University Eye Clinic and are offered multidisciplinary counseling.

This is the first Italian study to analyze non-syndromic and syndromic RP patients, including familial and sporadic cases. There have been only 3 studies examining the molecular epidemiology of specific forms of RP in Italy: a study on 43 adRP families (Ziviello et al., 2005), a characterization of 75 families with Usher syndrome (Vozzi et al., 2011), and a report on 15 familial cases of XL-RP (Miano et al., 1999).

We carried out mutation analysis in 41 index patients with non-syndromic RP and different inheritance patterns and 15 probands with syndromic RP. We identified causative mutations in 19 of the 56 probands (33.9\%), detecting 22 different heterozygous mutations, 12 of which are new variants not previously reported.

In patients with non-syndromic RP, molecular screening was positive for 10 of 41 probands (24.4\%). The results for adRP cases showed similar test positivity to that (27.9\%) reported by Ziviello et al. (2005); however, some of the genes analyzed in their panel were 
different from those analyzed in our study (including $N R L, C R X$, and PRPF8). A causative variant was found in 2 of 9 adRP patients $(22.2 \%)$. As discussed above, the p.Cys 165 Arg mutation in the PRPH2 gene, previously reported by Simonelli et al. (2007), and the new variant detected in the $R H O$ gene (p.Gly182 Val) were classified as pathogenic.

Molecular screening of 6 arRP probands and 18 sporadic cases led to the identification of heterozygous variants in the EYS and $R H O$ genes. Five new sequence variants in the EYS gene were detected in our screening. As described above, 4 of these novel variants (p.Thr3100Lysfs*26, c.-459C > T, p.Cys2139Arg, and p.Cys2927Arg) were classified as pathogenic, while the p.Pro1631Ser variant identified in sporadic proband P24 was classified as neutral based on bioinformatic analysis. Functional studies will be performed to assess the effect of all newly identified mutations on protein function.

The p.Pro347Leu missense mutation in $R H O$, which we detected in a sporadic RP patient initially showing RP symptoms at the age of 20 years, was previously associated with adRP in the Italian population (Dryja et al., 1990; Ziviello et al., 2005). The association of this mutation with early onset of disease at 12-23 years of age (Ziviello et al., 2005; Simonelli et al., 2007; Kim et al., 2011) was confirmed in our study.

In agreement with a previous study, we found that EYS was mainly associated with sporadic and recessive forms of non-syndromic RP (Abd El-Aziz et al., 2008). Notably, this is the first study identifying the association between EYS mutations and non-syndromic RP forms in the Italian population.

Genetic test positivity was significant in index patients with XL-RP who were analyzed for $R P 2$ and $R P G R$ point mutations (4 of 8 positive cases); we also screened the ORF15 region of $R P G R$, a unique terminal exon present in the alternatively spliced transcript, which is the predominant form of $R P G R$ in the retina (Vervoort et al., 2000; Pusch et al., 2002; Sharon et al., 2003). Three RPGR mutations were detected in ORF15 (p.Glu802Glyfs*32, p.Glu675*, and p.Asp656Valfs*41) and 1 was found in $R P G R$ exon 5 (p.Gly148Val). The latter variant and the 2 frameshift mutations affecting $R P G R$-ORF15 were confirmed to segregate with the disease phenotype in other family members available for genetic testing. As expected, patients with $\mathrm{XL}-\mathrm{RP}$ due to mutation in the $R P G R$ gene were found to have a severe form of inherited retinal degeneration, characterized by childhood onset, rapid progression, and early macular involvement with decreased visual acuity, leading to significant vision loss before the 4th decade (P35); various degrees of retinal dysfunction were described in heterozygous carrier females.

A previous study examined 15 Italian families with XL-RP (Miano et al., 1999), but the ORF15 region, which was identified in a later study (Vervoort et al., 2000), was not screened. We therefore report the results of molecular screening of the ORF15 exon in Italian families for the first time. Our data agrees with the results of international studies indicating that $R P G R$ ORF15 mutations are responsible for 60-80\% of XL-RP cases (Vervoort et al., 2000).

Genetic test positivity for non-syndromic RP patients (24.4\%) detected in the present study agreed with the results of previous studies. The significant proportion of negative test results may have been observed because of the high clinical and genetic heterogeneity of RP, which is reflected by the number of underlying gene defects. Moreover, the prioritization of genes for analysis by Sanger sequencing is limited by the lack of a specific correlation between particular ophthalmological characteristics and genetic subtypes of RP, and molecular diagnostics is particularly challenging for examining isolated RP patients (Nájera et al., 1995; Hayakawa et al., 1997). 
Syndromic forms of RP, such as Usher2A and Bardet-Biedl syndrome, show some differences; the disease-causing genes identified thus far have been associated with an increased number of cases (USH2A with approximately $80 \%$ of patients with Usher syndrome type II; $B B S 1$ and $B B S 10$ with approximately $50 \%$ of Bardet-Biedl cases).

We identified causative mutations, including 2 known frameshift variants in the BBS10 gene, in 1 of 2 probands with Bardet-Biedl syndrome. Direct sequencing of exons 2-21 of the USH $2 A$ gene revealed heterozygous variants in 8 of 13 patients $(61.5 \%)$; the 2 causative mutations, expected to be associated with this recessive disorder, were only detected in 1 proband (P50). This is the first phase our screening, which did not include direct sequencing of exons 22-72 or detection of gross deletions. This protocol is based on previous studies (Aller et al., 2010; McGee et al., 2010). Analysis of the longer isoform of USH2A clearly increases the detection rate, and a second screening step is currently underway to complete the genetic testing of USH2A patients. We detected a total of 8 different mutations, 3 of which are new variants. A similar study performed in 39 Italian patients, using a chip for the most frequent variants identified in Caucasians, revealed USH2A causative mutations in $15.4 \%$ of cases (Vozzi et al., 2011). Among the mutations already associated with Usher type II, the c.2299del variant was found in $25 \%$ of patients ( 2 of 8 ); this variant is the most common mutation detected in Europe and the USA and may have a South-European origin (Eudy et al., 1998; Aller et al., 2010). The c.2299del frameshift variant (p.Glu767Serfs*21) and the nonsense mutation c.187C >T (p.Arg63*) identified in our patients were found to be the most frequently observed mutations in the Italian population and are described in the present study and in Vozzi et al. (2011).

In summary, our results help define the spectrum and frequency of mutations associated with different forms of syndromic and non-syndromic RP in Italian patients. Notably, 12 of the 22 putative causative variants identified were new mutations that have not been reported previously. Since confirmation of new mutations in other family members was not always possible, analysis of additional families and proper functional studies are needed to confirm the pathogenicity of some of these variants.

This is the first report in Italy including sporadic patients and probands with arRP and the association between EYS mutations and non-syndromic RP forms. The study also provides data regarding $R P G R$-ORF15 mutations in XL-RP patients, and thus important information about mutations causing RP in the Italian population. Understanding the unique pattern of frequent mutations in a specific ethnic group may facilitate the development of molecular diagnosis and personalized gene therapies, which have recently made considerable advances (Liu et al., 2011; Beltran et al., 2012), as well as facilitate the counseling of patients.

The percentage of RP mutations identified in the present and previous Italian studies, along with difficulties in the prioritization of genes for Sanger sequencing, suggest that innovative methodology such as next-generation sequencing (NGS) will be necessary for significantly improving the molecular diagnosis of RP. Applications of NGS for diagnosing heterogeneous disorders such as RP are supported by recent studies (Audo et al., 2012; O'Sullivan et al., 2012).

\section{ACKNOWLEDGMENTS}

We thank the patients and their families for taking part in the study, Dr. Francesca Massone for orthoptic collaboration, and Helen Ampt for reviewing the English language of the manuscript. 


\section{REFERENCES}

Abd El-Aziz MM, Barragan I, O’Driscoll CA, Goodstadt L, et al. (2008). EYS, encoding an ortholog of Drosophila spacemaker, is mutated in autosomal recessive retinitis pigmentosa. Nat. Genet. 40: 1285-1287.

Aller E, Larrieu L, Jaijo T, Baux D, et al. (2010). The USH2A c.2299delG mutation: dating its common origin in a Southern European population. Eur. J. Hum. Genet. 18: 788-793.

Audo I, Sahel JA, Mohand-Saïd S, Lancelot ME, et al. (2010). EYS is a major gene for rod-cone dystrophies in France. Hum. Mutat. 31: E1406-E1435.

Audo I, Bujakowska KM, Leveillard T, Mohand-Saïd S, et al. (2012). Development and application of a next-generationsequencing (NGS) approach to detect known and novel gene defects underlying retinal diseases. Orphanet. J. Rare Dis. 7: 8 .

Ayuso C, Garcia-Sandoval B, Najera C, Valverde D, et al. (1995). Retinitis pigmentosa in Spain. The Spanish multicentric and multidisciplinary group for research into retinitis pigmentosa. Clin. Genet. 48: 120-122.

Barragán I, Borrego S, Pieras JI, González-del PM, et al. (2010). Mutation spectrum of EYS in Spanish patients with autosomal recessive retinitis pigmentosa. Hum. Mutat. 31: E1772-E1800.

Beltran WA, Cideciyan AV, Lewin AS, Iwabe S, et al. (2012). Gene therapy rescues photoreceptor blindness in dogs and paves the way for treating human X-linked retinitis pigmentosa. Proc. Natl. Acad. Sci. U. S. A. 109: 2132-2137.

Chang S, Vaccarella L, Olatunji S, Cebulla C, et al. (2011). Diagnostic challenges in retinitis pigmentosa: genotypic multiplicity and phenotypic variability. Curr. Genomics 12: 267-275.

Daiger SP, Bowne SJ and Sullivan LS (2007). Perspective on genes and mutations causing retinitis pigmentosa. Arch. Ophthalmol. 125: 151-158.

Dryja TP, McGee TL, Reichel E, Hahn LB, et al. (1990). A point mutation of the rhodopsin gene in one form of retinitis pigmentosa. Nature 343: 364-366.

Eudy JD, Weston MD, Yao S, Hoover DM, et al. (1998). Mutation of a gene encoding a protein with extracellular matrix motifs in Usher syndrome type IIa. Science 280: 1753-1757.

Fahim AT, Daiger SP and Weleber RG (2013). Retinitis Pigmentosa Overview 2000 [Updated 2013 Mar 21]. In: GeneReviews ${ }^{\mathrm{TM}}$ [Internet] (Pagon RA, Adam MP, Bird TD, et al., eds.). Seattle (WA): University of Washington, Seattle, 1993-2013. Available at [http://www.ncbi.nlm.nih.gov/books/NBK1417/]. Accessed October 3, 2013.

Fossarello M, Serra A, Mansfield D, Wright A, et al (1993). Genetic and Epidemiological Study of Autosomal Dominant (ADRP) and Autosomal Recessive (ARRP) Retinitis Pigmentosa in Sardinia. In: Retinal Degeneration (Hollyfield JG, La Vail MM and Anderson RE, eds.). Plenum Press, New York, 79-90.

Hayakawa M, Fujiki K, Kanai A, Matsumura M, et al. (1997). Multicenter genetic study of retinitis pigmentosa in Japan: I. Genetic heterogeneity in typical retinitis pigmentosa. Jpn. J. Ophthalmol. 41: 1-6.

Kajiwara K, Berson EL and Dryja TP (1994). Digenic retinitis pigmentosa due to mutations at the unlinked peripherin/ RDS and ROM1 loci. Science 264: 1604-1608.

Katsanis N (2004). The oligogenic properties of Bardet-Biedl syndrome. Hum. Mol. Genet. 13 Spec No 1: R65-R71.

Keats BJ and Savas S (2004). Genetic heterogeneity in Usher syndrome. Am. J. Med. Genet. A. 130A: 13-16.

Kim KJ, Kim C, Bok J, Kim KS, et al. (2011). Spectrum of rhodopsin mutations in Korean patients with retinitis pigmentosa. Mol. Vis. 17: 844-853.

Kimberling WJ, Orten D and Pieke-Dahl S (2000). Genetic heterogeneity of Usher syndrome. Adv. Otorhinolaryngol. 56: 11-18.

Koenekoop RK, Loyer M, Hand CK, Al Mahdi H, et al. (2003). Novel RPGR mutations with distinct retinitis pigmentosa phenotypes in French-Canadian families. Am. J. Ophthalmol. 136: 678-687.

Liu MM, Tuo J and Chan CC (2011). Republished review: Gene therapy for ocular diseases. Postgrad. Med. J. 87: 487495.

Mansergh FC, Millington-Ward S, Kennan A, Kiang AS, et al. (1999). Retinitis pigmentosa and progressive sensorineural hearing loss caused by a C12258A mutation in the mitochondrial MTTS2 gene. Am. J. Hum. Genet. 64: 971-985.

McGee TL, Seyedahmadi BJ, Sweeney MO, Dryja TP, et al. (2010). Novel mutations in the long isoform of the USH2A gene in patients with Usher syndrome type II or non-syndromic retinitis pigmentosa. J. Med. Genet. 47: 499-506.

Miano MG, Testa F, Strazzullo M, Trujillo M, et al. (1999). Mutation analysis of the RPGR gene reveals novel mutations in south European patients with X-linked retinitis pigmentosa. Eur. J. Hum. Genet. 7: 687-694.

Nájera C, Millán JM, Beneyto M and Prieto F (1995). Epidemiology of retinitis pigmentosa in the Valencian community (Spain). Genet. Epidemiol. 12: 37-46.

Nakanishi H, Ohtsubo M, Iwasaki S, Hotta Y, et al. (2009). Identification of 11 novel mutations in USH2A among Japanese patients with Usher syndrome type 2. Clin. Genet. 76: 383-391. 
O'Sullivan J, Mullaney BG, Bhaskar SS, Dickerson JE, et al. (2012). A paradigm shift in the delivery of services for diagnosis of inherited retinal disease. J. Med. Genet. 49: 322-326.

Pagon RA and Daiger SP (2000). Retinitis Pigmentosa Overview 2000 [Updated 2005]. In: GeneReviews ${ }^{\mathrm{TM}}$ (Pagon RA, Bird TD, Dolan CR, et al., eds.). University of Washington, Seattle. Available at [http://www.ncbi.nlm.nih.gov/ books/NBK1417/]. Accessed September 9, 2013.

Parmeggiani F, Sato G, De Nadai K, Romano MR, et al. (2011). Clinical and rehabilitative management of retinitis pigmentosa: Up-to-Date. Curr. Genomics 12: 250-259.

Pusch CM, Broghammer M, Jurklies B, Besch D, et al. (2002). Ten novel ORF15 mutations confirm mutational hot spot in the RPGR gene in European patients with X-linked retinitis pigmentosa. Hum. Mutat. 20: 405.

RetNet (2013). The University of Texas Health Science Center, 1996-2013. Retinal Information Network. Available at [http://www.sph.uth.tmc.edu/RetNet/]. Accessed January 25, 2013.

Rivolta C, Sharon D, DeAngelis MM and Dryja TP (2002). Retinitis pigmentosa and allied diseases: numerous diseases, genes, and inheritance patterns. Hum. Mol. Genet. 11: 1219-1227.

Scheper GC, van der Knaap MS and Proud CG (2007). Translation matters: protein synthesis defects in inherited disease. Nat. Rev. Genet. 8: 711-723.

Sharon D, Sandberg MA, Rabe VW, Stillberger M, et al. (2003). RP2 and RPGR mutations and clinical correlations in patients with X-linked retinitis pigmentosa. Am. J. Hum. Genet. 73: 1131-1146.

Sheffield VC, Fishman GA, Beck JS, Kimura AE, et al. (1991). Identification of novel rhodopsin mutations associated with retinitis pigmentosa by GC-clamped denaturing gradient gel electrophoresis. Am. J. Hum. Genet. 49: 699-706.

Simonelli F, Testa F, Marini V, Interlandi E, et al. (2007). Intrafamilial clinical heterogeneity associated with a novel mutation of the retinal degeneration slow/peripherin gene. Ophthalmic Res. 39: 255-259.

Vervoort R, Lennon A, Bird AC, Tulloch B, et al. (2000). Mutational hot spot within a new RPGR exon in X-linked retinitis pigmentosa. Nat. Genet. 25: 462-466.

Vozzi D, Aaspollu A, Athanasakis E, Berto A, et al. (2011). Molecular epidemiology of Usher syndrome in Italy. Mol. Vis. 17: 1662-1668.

Waters AM and Beales PL (2003). Bardet-Biedl Syndrome. [Updated 2011] In: GeneReviews ${ }^{\mathrm{TM}}$ (Pagon RA, Bird TD, Dolan CR, et al., eds.). University of Washington, Seattle. Available at [http://www.ncbi.nlm.nih.gov/books/ NBK1363]. Accessed September 9, 2013.

Ziviello C, Simonelli F, Testa F, Anastasi M, et al. (2005). Molecular genetics of autosomal dominant retinitis pigmentosa (ADRP): a comprehensive study of 43 Italian families. J. Med. Genet. 42: e47. 